\title{
The Spectrum of Quantum Dots Film for UV CCD
}

\author{
Lin Jiang, ${ }^{1}$ Haojie Sun, ${ }^{2}$ Bang-lian Xu, ${ }^{1}$ Da-wei Zhang, ${ }^{1}$ Chun-xian Tao, ${ }^{1}$ \\ Yuan-shen Huang, ${ }^{1}$ Zheng-ji Ni, ${ }^{1}$ and Song-lin Zhuang ${ }^{1}$ \\ ${ }^{1}$ Shanghai Key Laboratory of Modern Optical System, School of Optical-Electrical and Computer Engineering, \\ Shanghai Publishing and Printing College, University of Shanghai for Science and Technology, Shanghai 200093, China \\ ${ }^{2}$ Shanghai Publishing and Printing College, Shanghai 200093, China
}

Correspondence should be addressed to Da-wei Zhang; dwzhang@usst.edu.cn

Received 10 April 2013; Accepted 3 June 2013

Academic Editor: M. Carmen Yebra-Biurrun

Copyright (C) 2013 Lin Jiang et al. This is an open access article distributed under the Creative Commons Attribution License, which permits unrestricted use, distribution, and reproduction in any medium, provided the original work is properly cited.

\begin{abstract}
A new kind of UV-responsive film with quantum dots (QDs) fabricated by a spin-coating method is proposed in this paper. In a previous study, the monolayer QDs film is deposited onto fused silica slides by the spin-coating way, which has some luminous problem. The introduction of composite QDs coating which adds PEDOT:PSS and poly-TPD films to the monolayer QDs film is found to have excellent performance. The reason can be that PEDOT:PSS and poly-TPD weaken the scattering and enhance the emitting of quantum dot fluorescence. The intensity of photoluminescence (PL) for composite QDs coating is dozens of times stronger than that for monolayer QDs film. Experiment results show that this composite coating has excellent fluorescent properties and emits a blue purple glow together a wide excitation spectrum field from $190 \mathrm{~nm}$ to $300 \mathrm{~nm}$. The spectrum of the composite coating matches accurately with the detected zone of CCD, which achieves an outstanding UV-responsive coating for conventional silicon-based image sensors.
\end{abstract}

\section{Introduction}

Silicon-based imaging detector CCD has been widely used in the scientific fields. But the typical CCD image sensors are not sensitive to ultraviolet (UV) radiation, because the penetration depth of UV photons can only reach about $2 \mathrm{~nm}$ in a polysilicon gate material with thickness of $1 \mu \mathrm{m}$ [1]. Therefore researchers from all over the world have quested the method of enhancing the UV response of the CCD. In order to enhance the UV response of CCD detectors, a kind of UVresponsive film has been coated on the surface of CCD, which could absorb the UV radiation and reemit it in the visible spectrum where photons can penetrate the gate material. This method is very useful since it does not require change the structure of the typical CCD to be changed, and the production engineering will be simplified [2]

The research was started in the UV-responsive film abroad since 1980s. According to the used materials, the conversion film can be divided into two categories-organic film and inorganic one. Organic conversion coating technology is relatively mature, with various products invented. However, this kind of film has a fatal weakness that the degradation of organic molecules in the UV radiation is very fast. In the light of illumination for $1 \mu \mathrm{W} / \mathrm{cm}^{2}$, the organic molecules degrade exponentially with the rate highly up to 3\% [3-5]. Moreover, because of the organic nature of organic molecules and their low melting and boiling points, stability of these films has also been in question.

Inorganic fluorescent material is usually composed by rare earth oxides and crystals. The crystal diameter of the inorganic fluorescent material is generally in the range of 1 to $150 \mu \mathrm{m}$. Although the life of the films produced via spincoating precipitation method is quite long, generally up to 5500 hours, there are still many defects in the film thickness and uniformity [6]. On the other hand, due to the advantages of quantum dots, such as long fluorescence lifetimes, wide excitation spectra, narrow emission spectra, and controllable emission wavelength by quantum dot size, inorganic quantum dots have been widely used in LEDs, biological detection and many other fields [7-11]. 


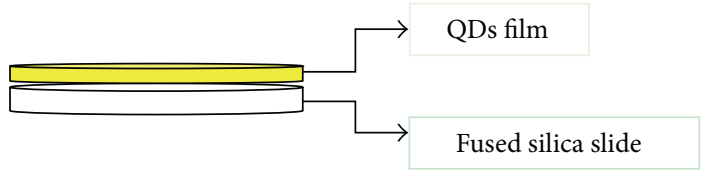

FIGURE 1: Structure of monolayer sample.

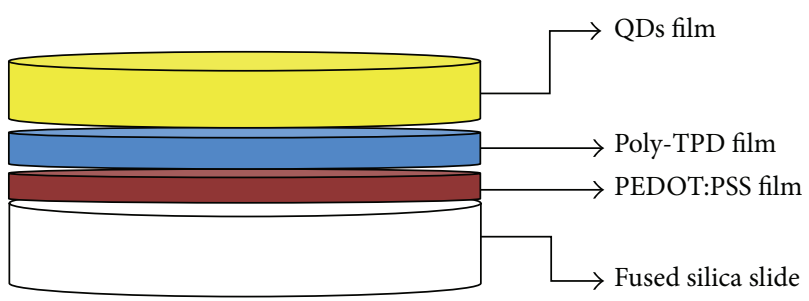

FIGURE 2: Structure of composite sample.

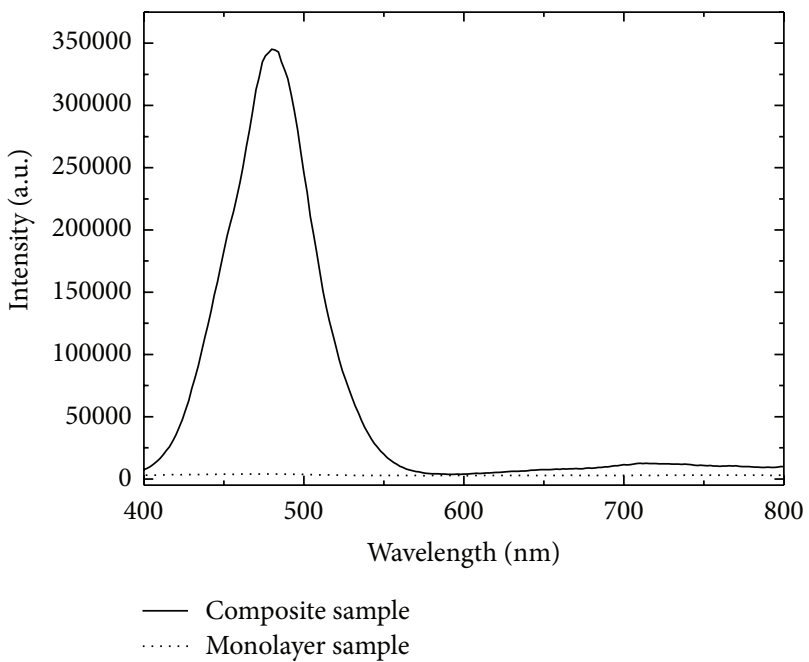

FIGURE 3: Photoluminescence emission spectra of composite sample (straight line) and monolayer sample (dotted line) at $190 \mathrm{~nm}$.

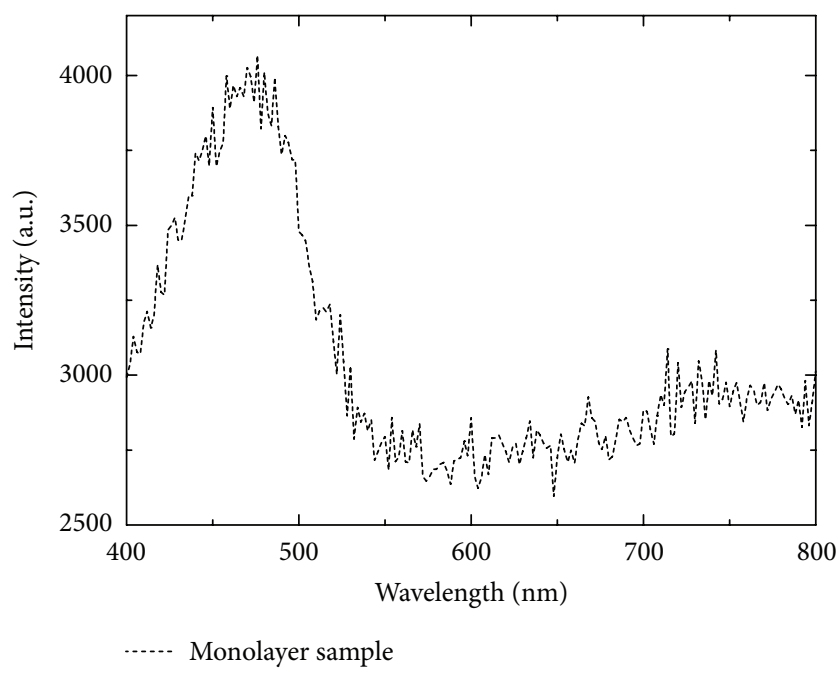

FIGURE 4: Photoluminescence emission spectra of monolayer sample at $190 \mathrm{~nm}$.

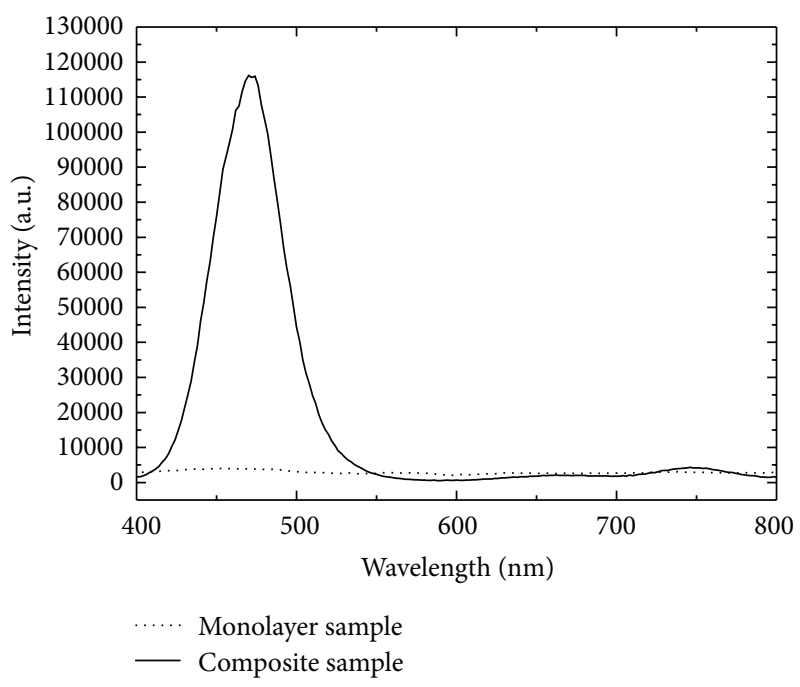

FIGURE 5: Photoluminescence emission spectra of composite sample (straight line) and monolayer sample (dotted line) at $250 \mathrm{~nm}$.

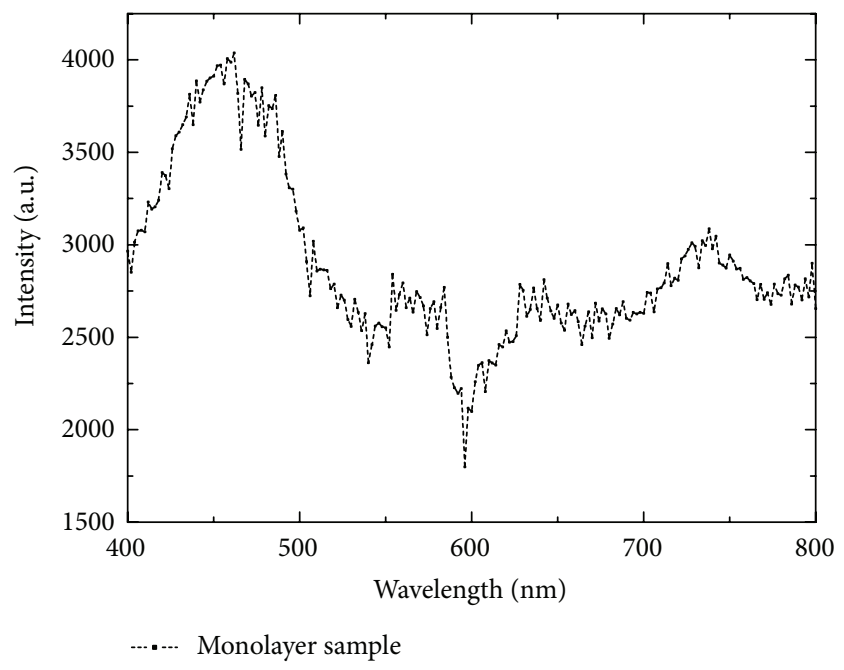

FIGURE 6: Photoluminescence emission spectra of monolayer sample at $250 \mathrm{~nm}$.

In this paper, a new kind of UV-responsive film with composite quantum dots (QDs) manufactured by a spin-coating method is reported. Compared to scintillators, QDs can achieve a similar function of wavelength conversion but present a more excellent fluorescent property on both intensity and lifetime. The composite coating was formed by adding the PEDOT:PSS and poly-TPD films under the QDs film which will weaken the scattering and enhance the emitting of 


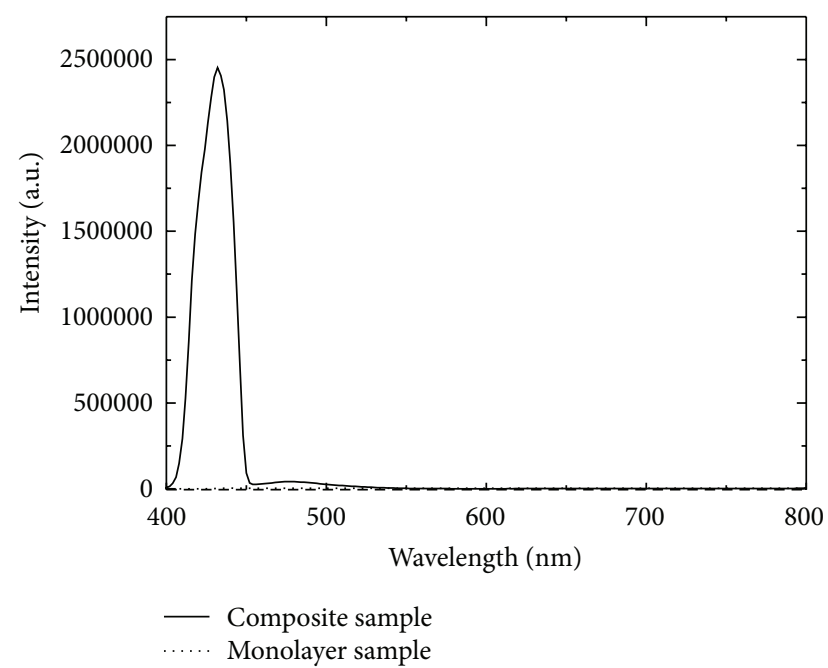

FIGURE 7: Photoluminescence emission spectra of composite sample (straight line) and monolayer sample (dotted line) at $300 \mathrm{~nm}$.

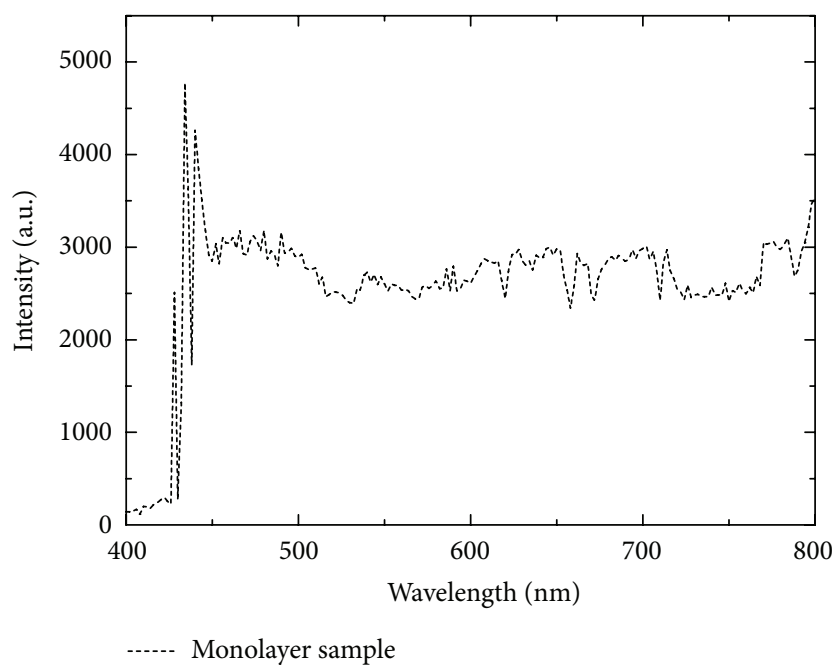

Figure 8: Photoluminescence emission spectra of monolayer sample at $300 \mathrm{~nm}$.

quantum dot fluorescence. In our experiment, it emitted an intensive blue purple glow and responded in a wide excitation spectrum field from $190 \mathrm{~nm}$ to $300 \mathrm{~nm}$, which complies well with the requirements of the CCD for UV detection.

\section{Materials and Methods}

The QDs used in this work are CdSe/ZnS with core-shell structure, which can increase the photostability of the QDs. The structures of monolayer sample and composite sample are shown in Figures 1 and 2, respectively.

The details of the experimental procedures are given in the following paragraphs.

For monolayer sample

(1) clean the fused silica slide in the deionized water, propanol, ethanol, and ethyl propanol successively and by ultrasonic clearing machine in the end;
TABLE 1: The center wavelengths and intensity of PL emission spectra for monolayer and composite samples.

\begin{tabular}{lccc}
\hline & $190 \mathrm{~nm}$ & $250 \mathrm{~nm}$ & $300 \mathrm{~nm}$ \\
\hline $\begin{array}{l}\text { Composite } \\
\text { QDs film }\end{array}$ & $480 \mathrm{~nm} / 345099$ & $470 \mathrm{~nm} / 116209$ & $432 \mathrm{~nm} / 2453072$ \\
$\begin{array}{l}\text { Monolayer } \\
\text { QDs film }\end{array}$ & $480 \mathrm{~nm} / 4011$ & $468 \mathrm{~nm} / 3895$ & $434 \mathrm{~nm} / 4772$ \\
\hline
\end{tabular}

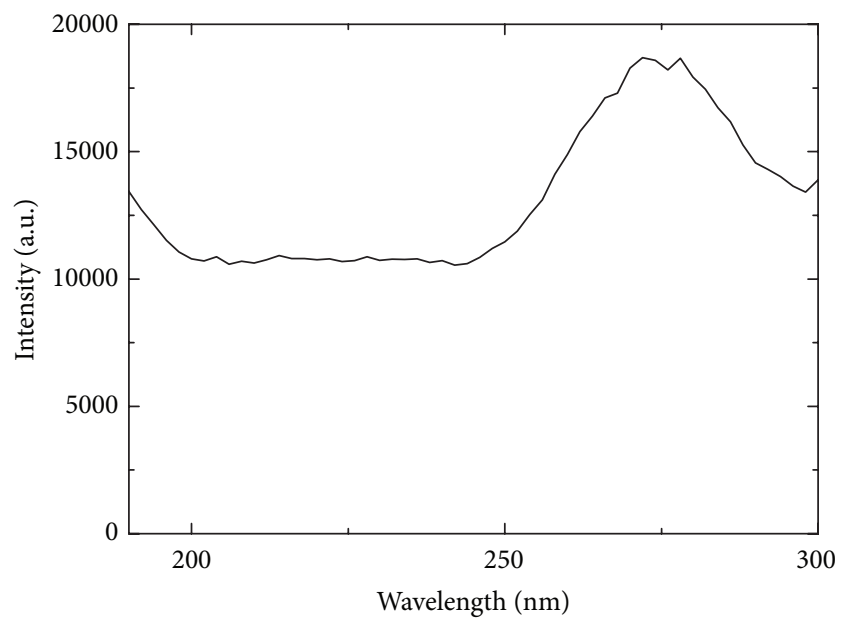

Figure 9: Photoluminescence excitation spectra of composite sample emitted at $480 \mathrm{~nm}$.

(2) deposit QDs film onto the fused silica slides by the spin-coating way, and then keep the sample at a temperature of 80 degrees for 20 minutes.

For composite sample:

(1) repeat step (1) of monolayer sample;

(2) make PEDOT:PSS film spin onto the fused silica slide, and then keep it at a temperature of 150 degrees for 30 minutes;

(3) deposit poly-TPD film onto PEDOT:PSS film by the method of spin coating, and then keep the sample at a temperature of 120 degrees for 30 minutes;

(4) repeat step (2) of monolayer sample.

\section{Results and Discussion}

The PL spectra of monolayer and composite samples operating at different wavelengths are shown in Figures 3, 4, 5, 6, 7, and 8 , with the detailed data displayed in Table 1 .

As shown in Figure 3, the PL intensity is significantly enhanced if PEDOT:PSS and poly-TPD are added. Besides, it can be seen from Table 1 that when the light of $190 \mathrm{~nm}$ is used to excite the samples, the emission center wavelengths of the two samples are both at $480 \mathrm{~nm}$. However, the PL intensity of the composite sample which added PEDOT:PSS and poly-TPD is 86.04 times larger than the monolayer sample. Viewing Figures 5-8 and Table 1, we can find that the results are almost the same. It can be calculated from Table 1 that at 


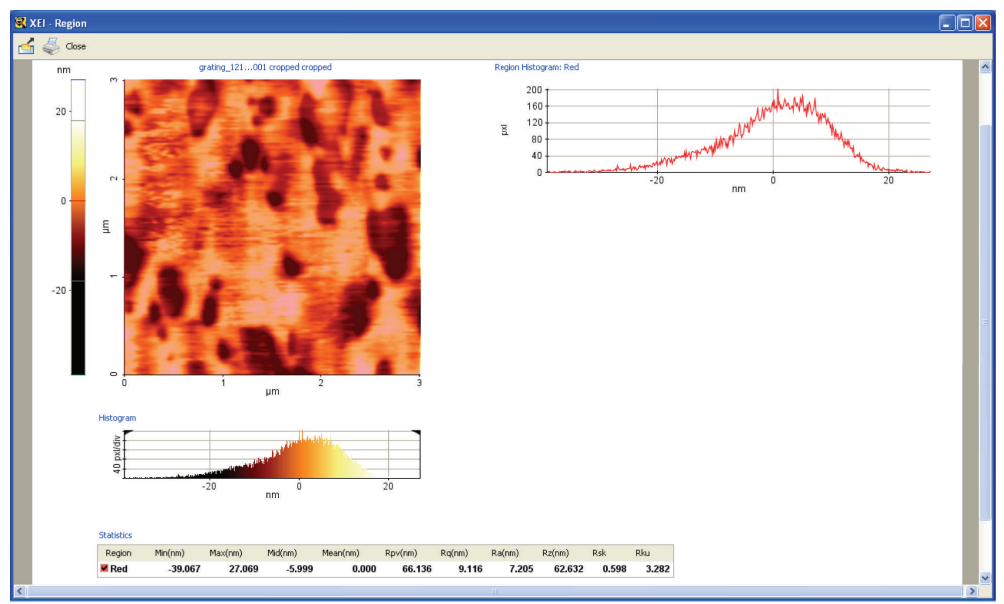

FIGURE 10: AFM image of monolayer sample.

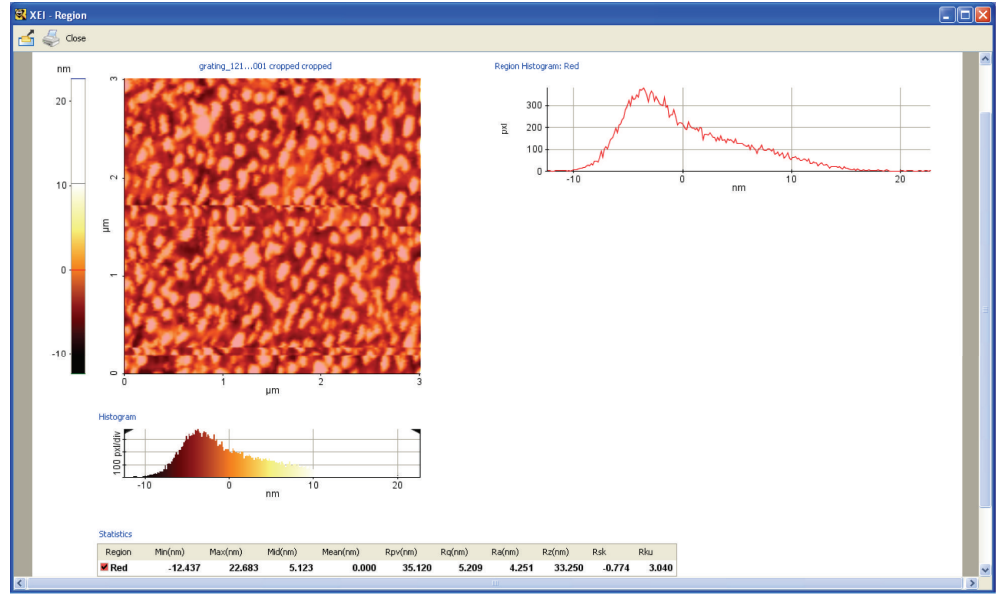

FIGURE 11: AFM image of composite sample.

$250 \mathrm{~nm}$, the peak of the composite sample is 29.84 times bigger than that of the monolayer sample, while it is 514.06 times at $300 \mathrm{~nm}$.

According to the above discussion, we can get the conclusion that PEDOT:PSS and poly-TPD can greatly enhance the PL intensity without affecting the position of emission peaks and therefore achieve the purpose of transformation of the wavelength - that is to say, the UV light is converted to visible light to meet the demand for UV-responsive film. By analysis, the reasons should be the presence of PEDOT:PSS and polyTPD, which weaken the scattering and enhance the emitting of quantum dot fluorescence [12-18].

The photoluminescence excitation spectra of the composite coating emitted at $480 \mathrm{~nm}$ are shown in Figure 9; we can see that the composite sample has a wide excitation spectrum in the UV wavelengths range.

Atomic Force Microscope (AFM) was used to examine the morphology of coating surfaces in this case [19-21]. The AFM images of monolayer sample and composite sample are shown in Figures 10 and 11, and the detail data are given in
Table 2. From Figures 10 and 11 and Table 2, we can easily reach a conclusion that the composite sample has a more uniform and lower roughness surface, which will reduce the light scattering and enhance the fluorescence emission.

\section{Conclusions}

This paper creatively presents the use of QDs coating as UVresponsive film and proposes a kind of effective composite coating. The results show that the composite QDs coating which added the PEDOT:PSS and poly-TPD films can greatly enhance the fluorescence intensity compared to the monolayer QDs film. The reasons should be attributed to the presence of PEDOT:PSS and poly-TPD, which weaken the scattering and enhance the emitting of quantum dot fluorescence. The composite coating can convert the UV light band of 190$300 \mathrm{~nm}$ into the visible light band of $400-500 \mathrm{~nm}$, and its emission spectrum lies in the CCD response band for a wide excitation spectrum; all of these characteristics just comply with the requirements of the CCD for UV detection. 
TABLE 2: AFM test data of monolayer and composite samples.

\begin{tabular}{lcc}
\hline & $\mathrm{Rq}(\mathrm{nm})$ & $\mathrm{Ra}(\mathrm{nm})$ \\
\hline Monolayer QDS film & 9.116 & 7.205 \\
Composite QDS film & 5.209 & 4.251 \\
\hline
\end{tabular}

\section{Acknowledgments}

This work was partially supported by the National Natural Science Foundation of China (61176085, 11105149), the National Key Technologies R\&D Program (2011BAF02B04), the National Science Instrument Important Project (2011YQ15004003), Dawn Program of Shanghai Education Commission (11SG44), Shanghai Ultraprecision Optical Manufacture and Testing Center programs from Shanghai Committee of Science \& Technology (11DZ2290301), and the Leading Academic Discipline Project of Shanghai Municipal Government (S30502).

\section{References}

[1] W. A. R. Franks, M. J. Kiik, and A. Nathan, "UV-responsive CCD image sensors with enhanced inorganic phosphor coatings," IEEE Transactions on Electron Devices, vol. 50, no. 2, pp. 352-358, 2003.

[2] G. Naletto, G. Tondello, G. Bonanno, R. Di Benedetto, and S. Scuderi, "Response analysis in the 300- to 2500-(angstrom) spectral range of ultraviolet-enhanced charge-coupled devices," Optical Engineering, vol. 33, no. 8, pp. 2544-2552, 1994.

[3] D. W. Zhang, X. Tian, Y. S. Huang, Z. J. Ni, and S. L. Zhuang, "Preparation and spectral characterization of Lumogen coatings for UV-responsive CCD image sensors," Spectroscopy and Spectral Analysis, vol. 30, no. 5, pp. 1171-1174, 2010.

[4] A. M. Damento, A. A. Barcellos, and V. W. Schempp, "Stability of lumogen films on CCDs," in Charge-Coupled Devices and Solid State Optical Sensors V, vol. 2415 of Proceedings of SPIE, pp. 204210, 1995.

[5] A. Deslandesa, A. B. Wedding, S. R. Clarke, J. G. Matisons, and J. S. Quinton, "Characterisation of PVD Lumogen films for wavelength conversion applications," in Smart Structures, Devices, and Systems II, vol. 5649 of Proceedings of SPIE, pp. 616-626, 2005.

[6] W. A. R. Franks, M. J. Kiik, and A. Nathan, "Inorganic phosphor coatings for UV-responsive CCD image sensors," in Sensors and Camera Systems for Scientific, Industrial, and Digital Photography Applications, vol. 3965 of Proceedings of SPIE, pp. 33-41, 2000.

[7] H. Huang, A. Dorn, G. P. Nair, V. Bulović, and M. G. Bawendi, "Bias-induced photoluminescence quenching of single colloidal quantum dots embedded in organic semiconductors," Nano Letters, vol. 7, no. 12, pp. 3781-3786, 2007.

[8] B. Tomlin and Y. Wang, "Pricing and operational recourse in coproduction systems," Management Science, vol. 54, no. 3, pp. 522-537, 2008.

[9] L. A. DeLouise and H. Ouyang, "Photoinduced fluorescence enhancement and energy transfer effects of quantum dots porous silicon," Physica Status Solidi C, vol. 6, no. 7, pp. 1729-1735, 2009.

[10] P. O. Anikeeva, C. F. Madigan, S. A. Coe-Sullivan, J. S. Steckel, M. G. Bawendi, and V. Bulović, "Photoluminescence of CdSe/
$\mathrm{ZnS}$ core/shell quantum dots enhanced by energy transfer from a phosphorescent donor," Chemical Physics Letters, vol. 424, no. 1-3, pp. 120-125, 2006.

[11] B. Ullrich, X. Y. Xiao, and G. J. Brown, "Photoluminescence of $\mathrm{PbS}$ quantum dots on semi-insulating GaAs," Journal of Applied Physics, vol. 108, no. 1, Article ID 013525, 2010.

[12] J. Yuan, W. Guo, and E. Wang, "Utilizing a CdTe quantum dotsenzyme hybrid system for the determination of both phenolic compounds and hydrogen peroxide," Analytical Chemistry, vol. 80, no. 4, pp. 1141-1145, 2008.

[13] J. S. Biteen, N. S. Lewis, H. A. Atwater, H. Mertens, and A. Polman, "Spectral tuning of plasmon-enhanced silicon quantum dot luminescence," Applied Physics Letters, vol. 88, no. 13, Article ID 131109, 3 pages, 2006.

[14] B. Liang, A. Lin, N. Pavarelli et al., "GaSb/GaAs type-II quantum dots grown by droplet epitaxy," Nanotechnology, vol. 20, no. 45, Article ID 455604, 2009.

[15] M. Winger, T. Volz, G. Tarel et al., "Explanation of photon correlations in the far-off-resonance optical emission from a quantum-dot-cavity system," Physical Review Letters, vol. 103, no. 20, Article ID 207403, 2009.

[16] I. L. Medintz, L. Berti, T. Pons et al., "A reactive peptidic linker for self-assembling hybrid quantum dot-DNA bioconjugates," Nano Letters, vol. 7, no. 6, pp. 1741-1748, 2007.

[17] Y. Zhang, L. Mi, P. N. Wang et al., "Photoluminescence decay dynamics of ThiolCapped CdTe quantum dots in living cells under microexcitation," Small, vol. 4, no. 6, pp. 777-780, 2008.

[18] M. Haridas, L. N. Tripathi, and J. K. Basu, "Photoluminescence enhancement and quenching in metal-semiconductor quantum dot hybrid arrays," Applied Physics Letters, vol. 98, no. 6, Article ID 063305, 2011.

[19] Z. Zhao, T. M. Dansereau, M. A. Petrukhina, and M. A. Carpenter, "Nanopore-array-dispersed semiconductor quantum dots as nanosensors for gas detection," Applied Physics Letters, vol. 97, no. 11, Article ID 113105, 2010.

[20] B. Ullrich, X. Y. Xiao, and G. J. Brown, "Photoluminescence of $\mathrm{PbS}$ quantum dots on semi-insulating GaAs," Journal of Applied Physics, vol. 108, no. 1, Article ID 013525, 2010.

[21] D. Richter, R. Roßbach, W. M. Schulz et al., "Low-density InP quantum dots embedded in Ga0.51 In0.49 P with high optical quality realized by a strain inducing layer," Applied Physics Letters, vol. 97, no. 6, Article ID 063107, 2010. 

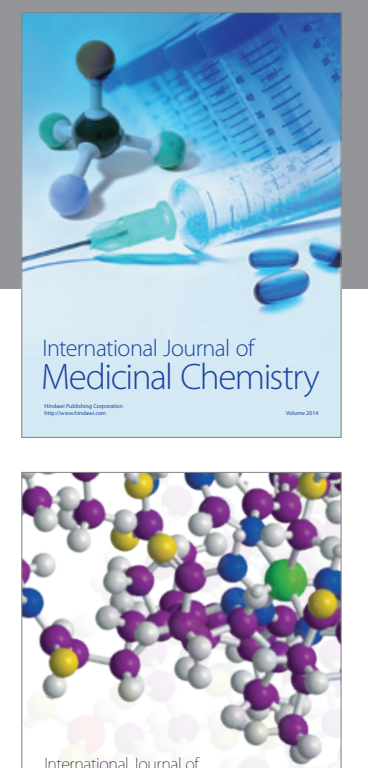

\section{Carbohydrate} Chemistry

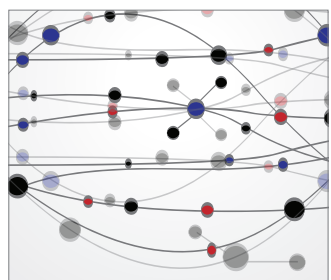

The Scientific World Journal
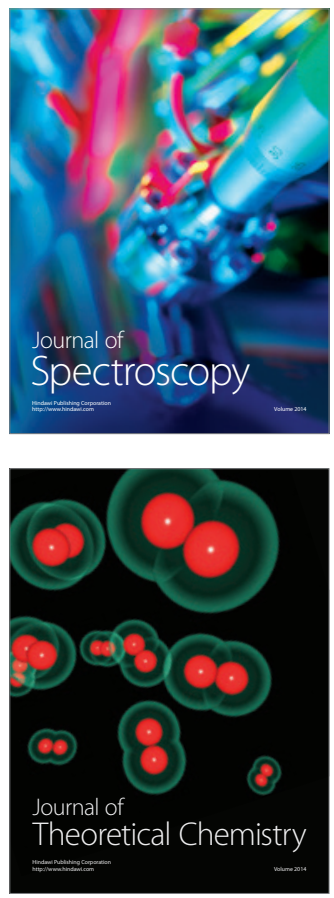
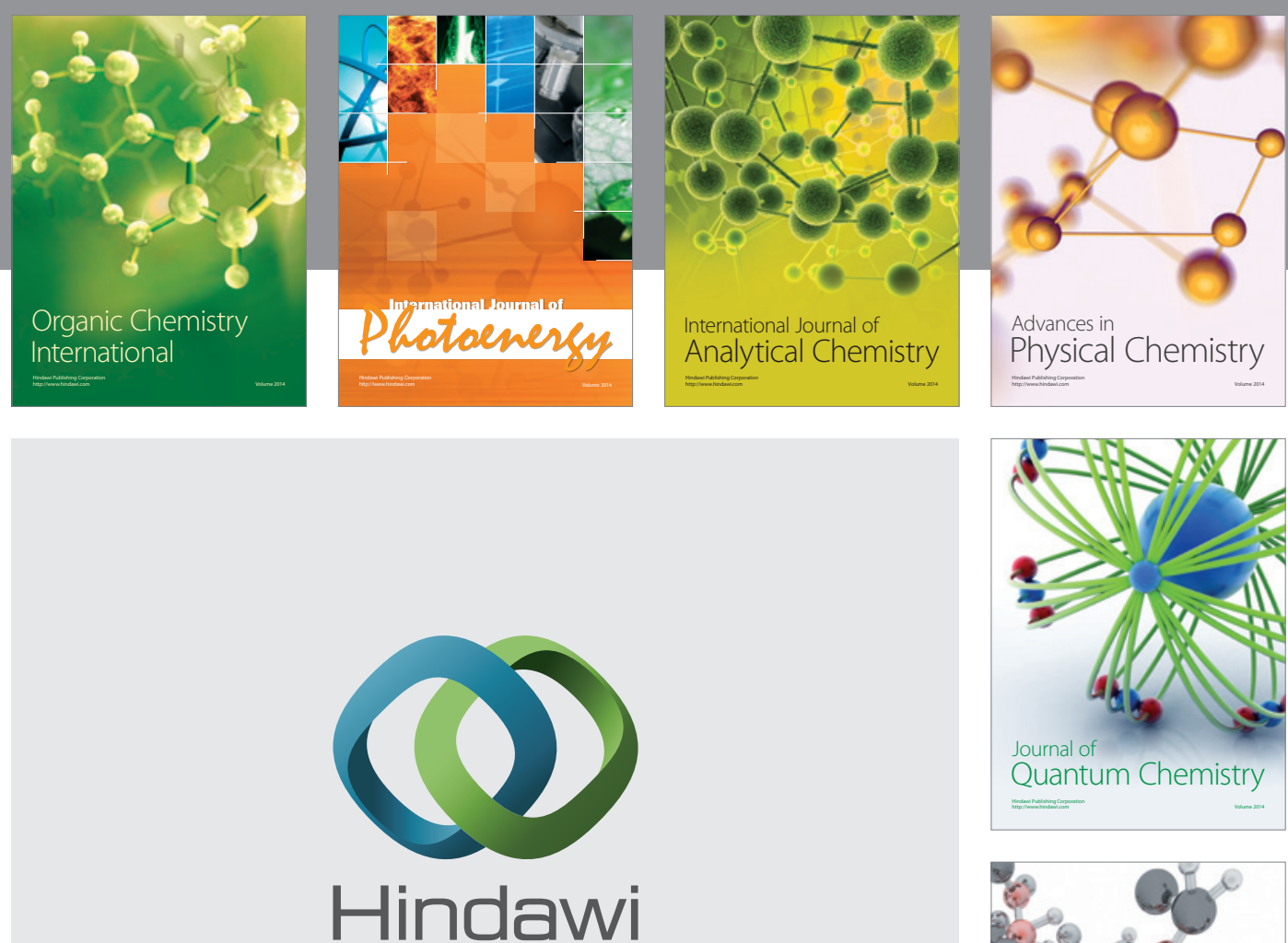

Submit your manuscripts at

http://www.hindawi.com

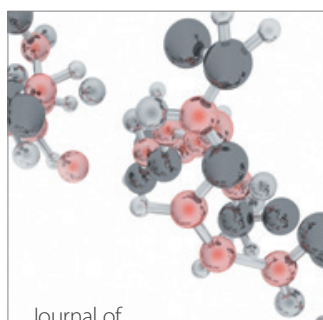

Analytical Methods

in Chemistry

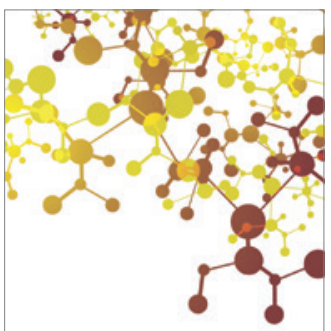

Journal of

Applied Chemistry

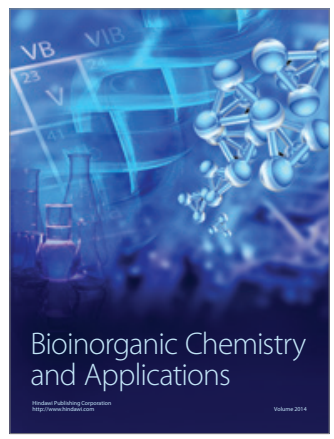

Inorganic Chemistry
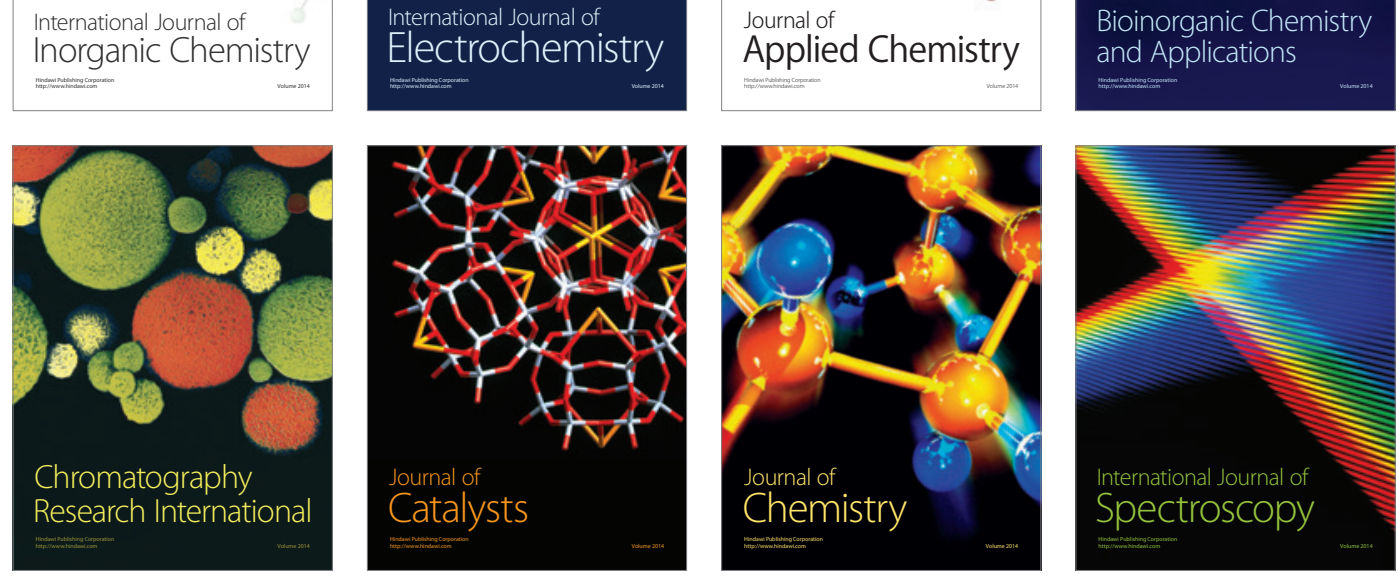